08

\title{
Влияние барьерных слоев бериллия на свойства многослойных зеркал Mo/Si
}

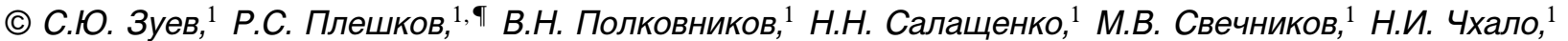 \\ F. Schäfers, ${ }^{2}$ M.G. Sertsu, ${ }^{2}$ A. Sokolov ${ }^{2}$ \\ ${ }^{1}$ Институт фризики микроструктур РАН, \\ 603087 Нижний Новгород, Россия \\ ${ }^{2}$ Helmholtz-Zentrum Berlin, \\ D-12489 Berlin, Germany \\ e-mail: pleshkov@ipmras.ru
}

Поступило в Редакцию 28 марта 2019 г.

В окончательной редакции 28 марта 2019 г.

Принято к публикации 15 апреля 2019 г.

\begin{abstract}
Приведены результаты исследований многослойных зеркал $\mathrm{Mo} / \mathrm{Si}$ с межслоевыми прослойками $\mathrm{B}_{4} \mathrm{C}$ и Ве в окрестности длины волны $13.5 \mathrm{~nm}$. Показано, что четырехкомпонентное многослойное зеркало типа $\mathrm{Mo} / \mathrm{Be} / \mathrm{Si} / \mathrm{B}_{4} \mathrm{C}$ по коэффициенту отражения превосходит на $2 \%$ зеркало $\mathrm{Mo} / \mathrm{Si}$ и на $1.3 \%$ зеркало $\mathrm{Mo} / \mathrm{Si} / \mathrm{B}_{4} \mathrm{C}$. Также это зеркало обеспечивает наибольшую спектральную полосу пропускания на полувысоте $\left(\Delta \lambda_{1 / 2}=0.535 \mathrm{~nm}\right)$. Дано объяснение этим эффектам.
\end{abstract}

Ключевые слова: рентгеновское излучение, многослойные зеркала, магнетронное распыление.

DOI: 10.21883/JTF.2019.11.48344.130-19

\section{Введение}

Интерес к многослойным зеркалам на основе молибдена и кремния во многом вызван развитием технологии проекционной литографии, а также связан с переходом от литографии глубокого ультрафиолета к литографии экстремального ультрафиолета (с рабочей длиной волны $13.5 \mathrm{~nm})$. В первую очередь здесь выделяется задача, связанная с необходимостью максимизации отражения в рассматриваемой спектральной области. С учетом многократного (10 раз и больше) отражения излучения при прохождении через оптическую систему литографической установки коэффициент отражения становится критически важной величиной. Увеличение его даже на относительно небольшую величину $(1-2 \%)$ может привести к существенному экономическому эффекту.

В работах, посвященных Mo/Si-зеркалам, показано, что их пиковый коэффициент отражения достигает $68 \%$ при теоретическом пределе 74\% [1-6]. Основной причиной отличия экспериментальных результатов от теоретического расчета является наличие протяженных переходных областей на границах раздела материалов структур. Отмечена также асимметрия переходных областей. В случае осаждения кремния на молибден ( $\mathrm{Si}-$ на-Mo) ширина оценивается как $0.5-0.6 \mathrm{~nm}$, в случае осаждения молибдена на кремний (Mo-на-Si) — как 1-1.2 nm [7].

Наиболее действенным способом увеличения коэффициентов отражения многослойных зеркал $\mathrm{Mo} / \mathrm{Si}$ признана методика барьерных слоев, заключающаяся в осаждении на поверхность молибдена и/или кремния третьих материалов. Наиболее распространенные барьерные материалы - углерод и карбид бора $\mathrm{B}_{4} \mathrm{C}$. Главная задача - это получение более протяженной границы Mo-на-Si. B результате максимальный коэффициент отражения для структур типа $\mathrm{Mo} / \mathrm{B}_{4} \mathrm{C} / \mathrm{Si} / \mathrm{B}_{4} \mathrm{C}$ и $\mathrm{Mo} / \mathrm{Si} / \mathrm{C}$ составил $70.15 \%$ на длине волны $13.5 \mathrm{~nm}$ [8].

B [9] сообщается о сглаживании границы Mo-на-Si до $0.6 \mathrm{~nm}$ при внедрении в систему $\mathrm{Mo} / \mathrm{Si}$ бериллия в качестве третьего материала. Порядок материалов от подложки к поверхности: молибден, бериллий, кремний $(\mathrm{Mo} / \mathrm{Be} / \mathrm{Si})$. При этом границы Be-на-Мо и $\mathrm{Si}-\mathrm{Ha}-\mathrm{Be}$ также обладают небольшой протяженностью (порядка $0.3 \mathrm{~nm})$. Экспериментально достигнуты коэффициенты отражения более $71 \%$ при $\lambda=13.5 \mathrm{~nm}$ и более $72 \%$ при $\lambda=12.9 \mathrm{~nm}$.

Здесь важно отметить, что толщина наиболее прозрачного материала Ве в этих экспериментах была довольно значительной $-1.5-2 \mathrm{~nm}$ (при общем периоде $6.9 \mathrm{~nm})$. В результате уменьшения общего поглощения структуры выросла глубина экстинкции. Полуширина пика отражения составила около $0.5 \mathrm{~nm}$. Для двухкомпонентной $\mathrm{Mo} / \mathrm{Si}$-структуры или для $\mathrm{Mo} / \mathrm{Si}$ с барьерными слоями карбида бора и углерода эта величина достигает $0.53 \mathrm{~nm}$. Уменьшение ширины пика отражения является следствием применения относительно „толстого“ слоя бериллия.

Важным выводом из рассмотренных работ является следующее утверждение. Сглаживание наиболее проблемной границы Mo-на-Si происходит и при осаждении $\mathrm{B}_{4} \mathrm{C}$ на поверхность кремния, но в случае осаждения Ве на поверхность молибдена остается невысокой шероховатость всех границ раздела. Кроме того, уменьшается общее поглощение.

Следующим шагом на пути повышения отражающей способности многослойных зеркал $\mathrm{Mo} / \mathrm{Si}$ должен стать синтез структуры типа $\mathrm{Mo} / \mathrm{Be} / \mathrm{Si} / \mathrm{B}_{4} \mathrm{C}$, причем толщина 


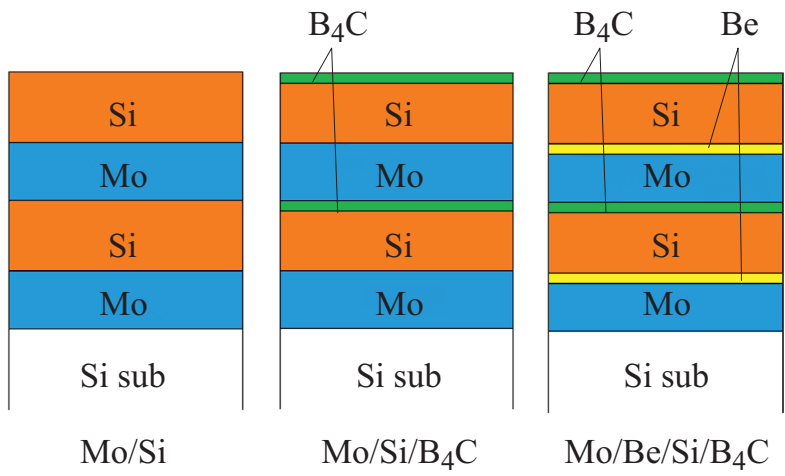

Рис. 1. Типы синтезированных многослойных структур.

слоя бериллия должна быть „барьерной“, т. е. составлять единицы ангстрем. Ожидается, что результатом этого станет сглаживание переходных областей между материалами без увеличения глубины экстинкции. В таких зеркалах можно ожидать повышения отражения без снижения ширины пика. Исследованию таких структур посвящена настоящая работа.

\section{Методика эксперимента}

Многослойные зеркала синтезировали методом магнетронного напыления при постоянном токе на установке, оснащенной шестью магнетронами планарного типа. Каждый магнетрон представляет собой источник с кольцевым разрядом. На поверхности расположена мишень распыляемого материала диаметром $150 \mathrm{~mm}$ и толщиной 5-6 mm. В качестве рабочей среды использовался высокочистый $(99.998 \%)$ газ аргон. Рабочее давление газа в технологическом процессе составляет $\sim 10^{-1} \mathrm{~Pa}$. Характерная скорость роста пленок $\sim 0.1-1 \mathrm{~nm} / \mathrm{s}$. Материалы, формирующие многослойные зеркала, осаждались на сверхгладкие (среднеквадратичная величина шероховатости $0.1-0.2 \mathrm{~nm}$ ) кремниевые подложки. Давление остаточных газов было не выше $10^{-4} \mathrm{~Pa}[10]$. Подробно об условиях напыления Весодержащих зеркал написано в [11].

В ходе экспериментов были синтезированы многослойные структуры трех типов: $\mathrm{Mo} / \mathrm{Si}, \mathrm{Mo} / \mathrm{Si} / \mathrm{B}_{4} \mathrm{C}$ и $\mathrm{Mo} / \mathrm{Be} / \mathrm{Si} / \mathrm{B}_{4} \mathrm{C}$. Схематически порядок материалов для двух периодов в зеркалах показан на рис. 1. Общее число периодов каждого типа зеркал равно 50.

Основные параметры многослойных зеркал, такие, как период, толщины индивидуальных слоев, межслоевые шероховатости, определялись по результатам малоугловой рентгеновской дифракции и рефлектометрии в окрестности $13.5 \mathrm{~nm}$.

Измерения методом малоугловой рентгеновской дифракции проводили в диапазоне углов падения излучения на образец $\theta=0^{\circ}-6^{\circ}$ с использованием четырехкристального высокоразрешающего дифрактометра PANalitycal X,Pert Pro $(\lambda=0.154 \mathrm{~nm})$.
В лаборатории измерения в окрестности длины волны $13.5 \mathrm{~nm}$ проводились на рефлектометре со спектрометром-монохроматором РСМ-500. Подробнее об измерениях можно посмотреть в [12]. Эти измерения дублировались синхротронными измерениями, выполненными на рефлектометре на оптической линии накопительного кольца BESSY-2 [13,14].

Изучались угловые (при фиксированной энергии фотонов) и спектральные (при фиксированном угле падения излучения) зависимости коэффициентов отражения многослойных зеркал. Периоды синтезированных образцов были близки друг другу. Это обеспечило корректное сравнение отражательных характеристик разных многослойных зеркал, поскольку максимумы коэффициентов отражения зеркал на спектральных зависимостях оказываются рядом, и можно не учитывать ни поляризацию рентгеновского излучения, ни шероховатости, влияние которой на коэффициент отражения сильно зависит от периода зеркала.

Параметры структур определялись методом одновременной подгонки кривых отражения, снятых на длине волны $0.154 \mathrm{~nm}$ и в окрестности $13.5 \mathrm{~nm}$, с использованием модели восстановления структурных параметров многослойных зеркал по данным рентгеновского отражения [15].

Пример подгонки результатов измерений зеркала $\mathrm{Mo} / \mathrm{Be} / \mathrm{Si} / \mathrm{B}_{4} \mathrm{C}$ приведен на рис. 2.

Нетрудно заметить хорошее совпадение экспериментальных и расчетных кривых одновременно на длинах волн $0.154 \mathrm{~nm}$ (рис. 2, $a$ ) и $13.5 \mathrm{~nm}$ (рис. 2, $b$ ). На рисунках символам отвечают экспериментальные данные дифрактометра PANalitycal X-Pert Pro и рефлектометра на оптической линии BESSY-II. Сплошные кривые результат моделирования (подгонки параметров модельной структуры).

\section{Результаты и их обсуждение}

На рис. 3 приведены данные малоугловой рентгеновской дифракции. Для наглядности здесь выделена область углов скольжения $4.4^{\circ}-6^{\circ}$, т. е. область 7-9-х дифракционных порядков.

Даже в логарифмическом масштабе хорошо видно, что наибольшими являются высоты пиков четырехкомпонентной структуры $\mathrm{Mo} / \mathrm{Be} / \mathrm{Si} / \mathrm{B}_{4} \mathrm{C}$, следующими по высоте - пики трехкомпонентной структуры $\mathrm{Mo} / \mathrm{Si} / \mathrm{B}_{4} \mathrm{C}$, а самыми низкими - у двухкомпонентного зеркала $\mathrm{Mo} / \mathrm{Si}$. Это служит косвенным свидетельством наибольшей протяженности переходных областей у $\mathrm{Mo} / \mathrm{Si}$ и наименьшей - у $\mathrm{Mo} / \mathrm{Be} / \mathrm{Si} / \mathrm{B}_{4} \mathrm{C}$.

По результатам измерений методом малоугловой рентгеновской дифракции и обработки данных измерений определены основные структурные параметры многослойных зеркал (табл. 1).

При сравнении структурных параметров для всех типов зеркал так же видна общая закономерность: $\sigma$ в случае многослойных зеркал с барьерными слоями заметно 

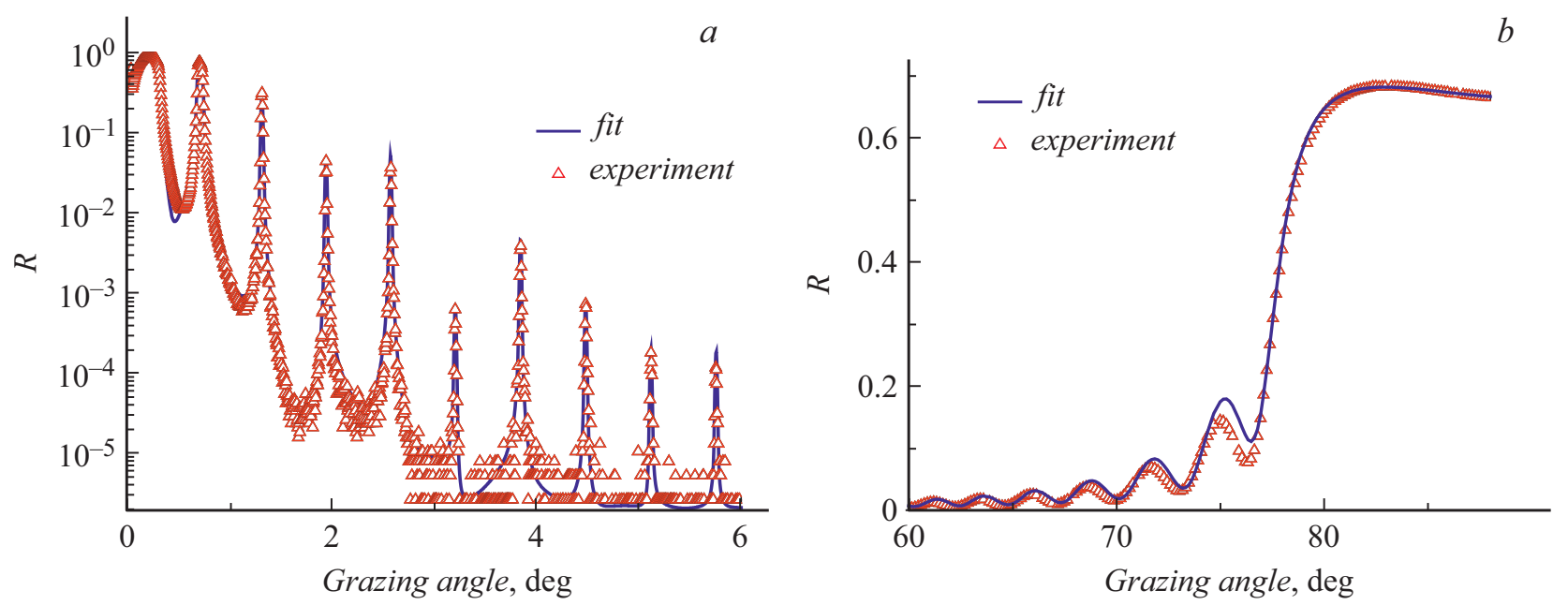

Рис. 2. Пример одновременной подгонки результатов измерений зеркала $\mathrm{Mo} / \mathrm{Be} / \mathrm{Si} / \mathrm{B}_{4} \mathrm{C}$, выполненной по данным малоугловой дифракции $(a)$ и рефлектометрии $(b)$. Символы - экспериментальные данные, сплошные кривые - результат моделирования.

Таблица 1. Основные структурные параметры исследуемых образцов $(\langle h(M)\rangle-$ средняя толщина пленки материала $M$ : Мо, $\mathrm{Si}$, $\mathrm{Be}, \mathrm{B}_{4} \mathrm{C}, \sigma$ - протяженность соответствующей переходной области)

\begin{tabular}{c|c|c|c}
\hline Структура & $\langle d\rangle, \mathrm{nm}$ & $\langle h(M)\rangle, \mathrm{nm}$ & $\sigma, \mathrm{nm}$ \\
\hline $\mathrm{Mo} / \mathrm{Si}$ & 6.9 & $h(\mathrm{Si})=4.15$ & $\sigma(\mathrm{Mo}-\mathrm{Ha}-\mathrm{Si})=0.9$ \\
& & $h(\mathrm{Mo})=2.75$ & $\sigma(\mathrm{Si}-\mathrm{Ha}-\mathrm{Mo})=0.45$ \\
\hline & & $h\left(\mathrm{~B}_{4} \mathrm{C}\right)=0.42$ & $h(\mathrm{Si})=3.74$ \\
& 6.91 & $h(\mathrm{Mo})=2.75$ & $\sigma\left(\mathrm{Mo}-\mathrm{Ha}-\mathrm{B}_{4} \mathrm{C}\right)=0.3$ \\
& & $h\left(\mathrm{~B}_{4} \mathrm{C}-\mathrm{Ha}_{-} \mathrm{Si}\right)=0.4$ \\
$\sigma(\mathrm{Si}-\mathrm{Ha}-\mathrm{Mo})=0.41$ \\
\hline
\end{tabular}

Таблица 2. Отражательные характеристики исследуемых образцов

\begin{tabular}{c|c|c|c|c}
\hline Структура & $\theta, \operatorname{deg}$ & $\lambda, \mathrm{nm}$ & $\Delta \lambda, \mathrm{nm}$ & $R, \%$ \\
\hline $\mathrm{Mo} / \mathrm{Si}$ & 85 & 13.45 & 0.505 & 66.5 \\
\hline $\mathrm{Mo} / \mathrm{Si} / \mathrm{B}_{4} \mathrm{C}$ & 83 & 13.45 & 0.52 & 67.1 \\
\hline $\mathrm{Mo} / \mathrm{Be} / \mathrm{Si} / \mathrm{B}_{4} \mathrm{C}$ & 83 & 13.45 & 0.535 & 68.4
\end{tabular}

меньше, чем в случае двухкомпонентной структуры $\mathrm{Mo} / \mathrm{Si}$. Это говорит о большей резкости межслоевых границ многослойных зеркал с барьерными слоями, и у $\mathrm{Mo} / \mathrm{Be} / \mathrm{Si} / \mathrm{B}_{4} \mathrm{C}$ эти границы оказываются наиболее резкими.

В результате многослойные зеркала $\mathrm{Mo} / \mathrm{Be} / \mathrm{Si} / \mathrm{B}_{4} \mathrm{C}$ имеют большее отражение в окрестности длины волны $13.5 \mathrm{~nm}$ (табл. 2).

На рис. 4 приведены экспериментально полученные спектральные зависимости коэффициента отражения для образцов с параметрами, соответствующими данным табл. 2.

Из рис. 4 видно, что многослойные зеркала $\mathrm{Mo} / \mathrm{Be} / \mathrm{Si} / \mathrm{B}_{4} \mathrm{C}$ демонстрируют большую отражательную эффективность. По высоте пика отражения четырехкомпонентная структура превосходит двухкомпонентную приблизительно на 2 абсолютных процента. При этом важно заметить, что ширина пика отражения на половине высоты для зеркала $\mathrm{Mo} / \mathrm{Be} / \mathrm{Si} / \mathrm{B}_{4} \mathrm{C}$ оказывается максимальной и составляет $0.535 \mathrm{~nm}$.

Таким образом, одновременное использование бериллия и карбида бора в качестве барьерных материалов действительно приводит к росту как пикового значения, так и полуширины коэффициента отражения многослойных зеркал на основе $\mathrm{Mo} / \mathrm{Si}$.

Следует оговориться, что продемонстрированные абсолютные значения коэффициентов отражения не достигают 70\%. Вероятно, это связано с тем, что изначально были выбраны неоптимальные индивидуальные толщины материалов. Тем не менее это не должно сказываться на соотношении коэффициентов отражения структур 


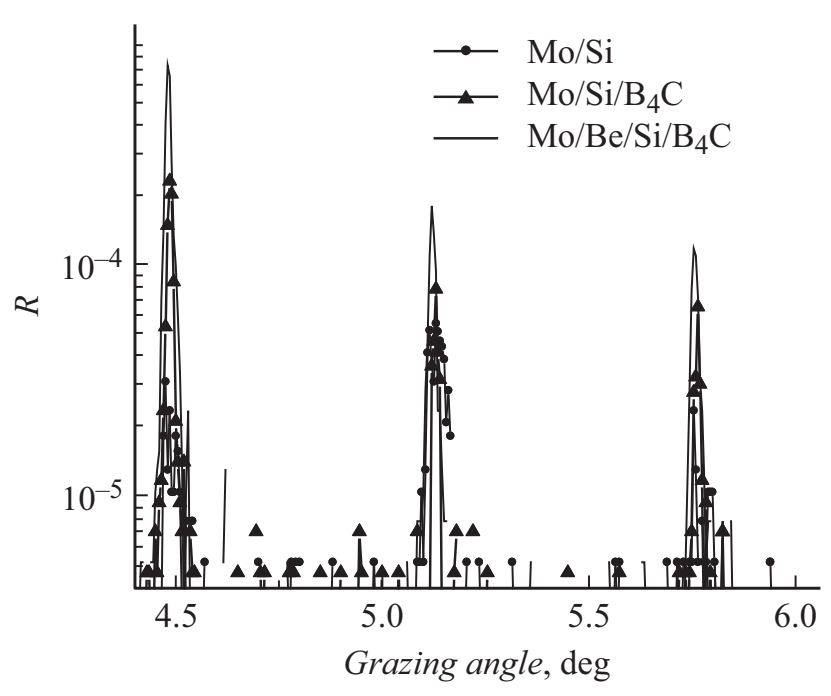

Pис. 3. Угловые зависимости коэффициентов отражения, измеренные в окрестности 7-9-х брэгговских пиков, зеркал $\mathrm{Mo} / \mathrm{Si}$ (круглые символы), Mo/Si/B 4 C (треугольные символы) и $\mathrm{Mo} / \mathrm{Be} / \mathrm{Si} / \mathrm{B}_{4} \mathrm{C}$ (сплошная кривая), длина волны $0.154 \mathrm{~nm}$.

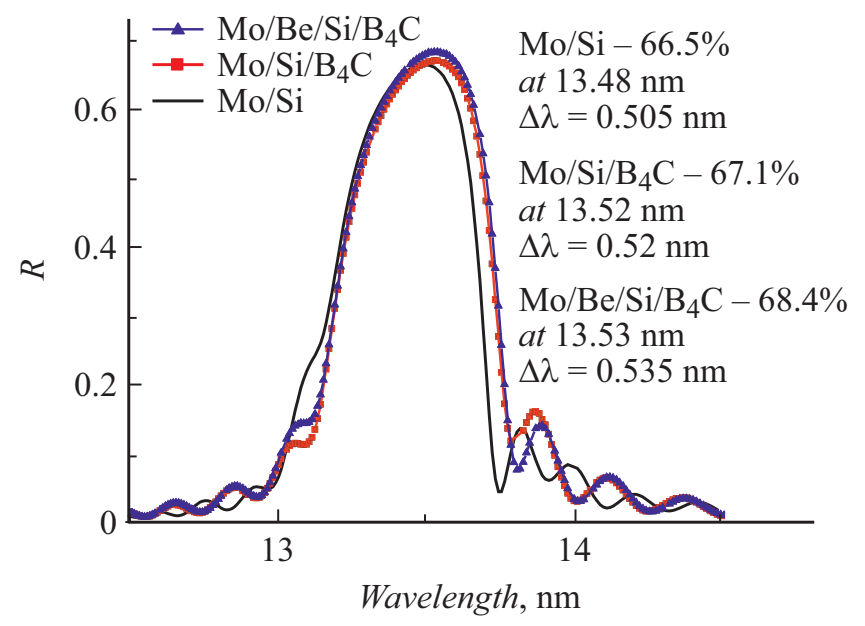

Рис. 4. Спектральные зависимости коэффициентов отражения зеркал $\mathrm{Mo} / \mathrm{Si}, \mathrm{Mo} / \mathrm{Si} / \mathrm{B}_{4} \mathrm{C}$ и $\mathrm{Mo} / \mathrm{Be} / \mathrm{Si} / \mathrm{B}_{4} \mathrm{C}$. Угол падения излучения составляет $2^{\circ}$ от нормали.

всех трех типов. Оптимизируя соотношение толщин материалов в элементарной ячейке многослойного зеркала, мы надеемся получить коэффициент отражения более $70 \%$ на длине волны $13.5 \mathrm{~nm}$.

\section{Финансирование работы}

Работа выполнена при поддержке грантов РФФИ 18-02-00588, 18-02-00173, 18-32-00173, 19-02-00081, 17-52-150006 и программы РАН 0035-2018-0011.

\section{Конфликт интересов}

Авторы заявляют, что у них нет конфликта интересов.

\section{Список литературы}

[1] Petford-Long A.K., Stearns M.B., Chang C.H. et al. // J. Appl. Phys. 1987. Vol. 61. N 4. P. 1422-1428.

[2] Rosen R.S., Vernon S.P., Stearns G. et al. // Appl. Opt. 1993. Vol. 32. N 34. P. 6975-6980.

[3] Slaughter J.M., Kearney P.A., Schulze D.W. et al. // Proc. SPIE. 1990. Vol. 1343. P. 73-82.

[4] Slaughter J.M., Schulze D.W., Hills C.R. et al. // J. Appl. Phys. 1994. Vol. 76. N 4. P. 2144-2156.

[5] Stearns D.G., Rosen R.S., Vernon S.P. // Appl. Opt. 1993. Vol. 32. N 34. P. $6952-6960$.

[6] Andreev S.S., Gaponova S.V., Gusev S.A. et al. // Thin Solid Films. 2002. Vol. 415. P. 123-132.

[7] Braun S., Mai H., Moss M. et al. // Jpn. J. Appl. Phys. 2002. Vol. 41. P. 4074-4081.

[8] Yakshin A.E., vande Kruijs R.W.E., Nedelcu I. et al. // Proc. SPIE. 2007. Vol. 6517. P. 65170I.

[9] Chkhalo N.I., Gusev S.A., Nechay A.N. et al. // Opt. Letters. 2017. Vol. 42. N 26. P. 5070-5073.

[10] Ахсахалян А.Д., Клюенков Е.Б., Лопатин А.Я. и др. // Поверхность. Рентген., синхротр. и нейтрон. исслед. 2017. № 1. C. 5-24.

[11] Chkhalo N.I., Pariev D.E., Polkovnikov V.N. et al. // Thin Solid Films. 2017. Vol. 631. P. 106-111.

[12] Andreev S.S., Akhsakhalyan A.D., Bibishkin M.A. et al. // Centr. Europ. J. Phys. 2003. Vol. 1. P. 191-209.

[13] Schäfers F., Bischoff P., Eggenstein F. et al. // J. Synchrotron Radiat. 2016. Vol. 23. P. 67-77.

[14] Sokolov A., Bischoff P., Eggenstein F. et al. // Rev. Sci. Instrum. 2016. Vol. 87. P. 052005.

[15] Svechnikov M., Pariev D., Nechay A. et al. // J. Appl. Cryst. 2017. Vol. 50. P. $1428-1440$. 